\title{
An Alternative Canonical Approach to the Ghost Problem in a Complexified Extension of the Pais-Uhlenbeck Oscillator ${ }^{\star}$
}

\author{
A. DÉCTOR ${ }^{\dagger}$, H.A. MORALES-TÉCOTL ${ }^{\dagger \ddagger}$, L.F. URRUTIA ${ }^{\dagger}$ and J.D. VERGARA ${ }^{\dagger}$ \\ $\dagger$ Instituto de Ciencias Nucleares, Universidad Nacional Autónoma de México, \\ A. Postal 70-543, México D.F., México \\ E-mail:dector@nucleares.unam.mx,urrutia@nucleares.unam.mx,vergara@nucleares.unam.mx \\ $¥$ Departamento de Física, Universidad Autónoma Metropolitana Iztapalapa, \\ San Rafael Atlixco 186, Col. Vicentina, CP 09340, México D.F., México \\ E-mail: hugo@xanum.uam.mx
}

Received November 14, 2008, in final form April 22, 2009; Published online May 05, 2009

doi:10.3842/SIGMA.2009.053

\begin{abstract}
Our purpose in this paper is to analyze the Pais-Uhlenbeck (PU) oscillator using complex canonical transformations. We show that starting from a Lagrangian approach we obtain a transformation that makes the extended PU oscillator, with unequal frequencies, to be equivalent to two standard second order oscillators which have the original number of degrees of freedom. Such extension is provided by adding a total time derivative to the PU Lagrangian together with a complexification of the original variables further subjected to reality conditions in order to maintain the required number of degrees of freedom. The analysis is accomplished at both the classical and quantum levels. Remarkably, at the quantum level the negative norm states are eliminated, as well as the problems of unbounded below energy and non-unitary time evolution. We illustrate the idea of our approach by eliminating the negative norm states in a complex oscillator. Next, we extend the procedure to the Pais-Uhlenbeck oscillator. The corresponding quantum propagators are calculated using Schwinger's quantum action principle. We also discuss the equal frequency case at the classical level.
\end{abstract}

Key words: quantum canonical transformations; higher order derivative models

2000 Mathematics Subject Classification: 70H15; 70H50; 81S10

\section{Introduction}

Systems with higher order time derivatives (HOTD) have been studied with increasing interest because they appear in many important physical problems. In first place these systems were considered to improve the divergent ultraviolet behavior of some quantum field theories [1]. However, their energy turned out not bounded from below [2], then involving ghosts and making the theory non-unitary [3]. In spite of such drawbacks, higher order derivative theories were studied to learn on their improved renormalization properties. Even a renormalizable higher order quantum gravity theory was advanced along these lines [4] and the unitarity of a lattice form was studied in [5]. See also $[6,7,8,9,10,11]$ and references therein for other examples. As for dealing with ghosts, attempts can be classified according to whether the approach is perturbative $[12,13,14]$ or not $[15,6,8]$, but a definite answer is yet to be found.

${ }^{\star}$ This paper is a contribution to the Proceedings of the VIIth Workshop "Quantum Physics with NonHermitian Operators" (June 29 - July 11, 2008, Benasque, Spain). The full collection is available at http://www.emis.de/journals/SIGMA/PHHQP2008.html 
Since single particle quantum mechanics can be seen, in the free field or weak coupling limit, as a mini-superspace sector of quantum field theory where the spatial degrees of freedom have been frozen, it is suggestive to test new ideas on HOTD theories, by using quantum mechanics as a laboratory. The model example to do so is the Pais-Uhlenbeck (PU) oscillator [2], which consists of a one dimensional harmonic oscillator Lagrangian plus a term quadratic in acceleration. A standard quantum treatment of its degrees of freedom gives rise to a spectrum not bounded from below since it consists of the difference of two quantum harmonic oscillators spectra [2]. An interesting solution to such difficulty has been recently proposed in $[16,17,18]$ (see also [19, 20, 21, 22] for a different approach) by finding a quantum transformation which gives its Hamiltonian a non-Hermitian, $\mathcal{P} \mathcal{T}$ symmetric form. In this approach a technique has been developed to obtain a physical inner product and thus a Hilbert space [23].

The aim of this paper is to show that it is possible to tackle the problem of quantizing an extension of the PU oscillator within a Lagrangian and a canonical ormulation, using complex canonical transformations and non-Hermitian variables together with reality conditions. This approach is motivated by a previous proposal to build complex canonical variables for nonperturbative quantum canonical general relativity [24, 25], which achieved the nontrivial task of making polynomial the constraints of general relativity, whereas the so called reality conditions to be fulfilled by the non-Hermitian variables were proposed to determine the inner product of Hilbert space. For canonical general relativity such reality conditions imply the metric of space and its rate of change are real quantities. Some effort was devoted to exploit these complex variables (see for instance [26, 27, 28]) however the use of real variables allowed finally for important progress $[29,30]$.

The paper is organized as follows. In Section 2 we review the essential aspects of the general construction with special emphasis on the boundary conditions. In Section 3 we use as a toy model a complexified harmonic oscillator [25]. Although this is not a higher-order derivative problem it exhibits negative norm states. We show that using the reality conditions we can eliminate them. Furthermore, we evaluate the Green's function of the system using the Schwinger quantum action principle and the path integral method, finding that both of these procedures lead to the same result. In Section 4 we combine the results of the previous sections in order to make a first attempt to extend the formalism to HOTD theories, successfully taking as an example the Pais-Uhlenbeck oscillator, which can be treated in a similar way as the complexified harmonic oscillator. In addition we perform the canonical analysis in an extended phase space in order to compare the cases of PU with equal and unequal frequencies. We observe that in the case of equal frequencies, the Lagrangian transformation induces a non canonical one which, at the quantum level, will lead to a non diagonalizable Hamiltonian. Finally, concluding remarks are presented in Section 5. Unless otherwise stated we use units in which $\hbar=1$.

\section{General considerations}

Let us consider a system whose Lagrangian contains up to the $n$-th order time derivative in a quadratic form. Written in normal coordinates this Lagrangian will have the form,

$$
L\left(x, \dot{x}, \ldots, x^{(n)}\right)=\sum_{i} \alpha_{i}\left(x^{(i)}\right)^{2}-V(x), \quad i=1, \ldots, n,
$$

with $x^{(k)}=\frac{d^{k} x}{d t^{k}}$ and the $\alpha_{i} \in \mathbb{R}$ being constants. We are assuming that under the transformation to normal coordinates we diagonalize the kinetic term and that the potential only depends on the coordinates. This Lagrangian yields an equation of motion of order $(2 n)$

$$
\sum_{k=0}^{n}(-1)^{k} \frac{d^{k}}{d t^{k}} \frac{\partial L}{\partial x^{(k)}}=0 .
$$


Assuming $x$ and hence $L$ to be real leads to the well known problems of the higher-order derivative models. So, as a first step in our extension we consider the analytic continuation of the variable $x$ to the complex plane. Next we assume that the Lagrangian (2.1) plus a total time derivative will be real. This certainly does not alter the classical equations of motion, but modifies the Hamiltonian formulation by the addition of new terms in the momenta. Now, the essential points of our procedure are the following two i) we assume that there exists a nonlocal transformation from the Lagrangian (2.1) plus a total time derivative to a second order real Lagrangian with $n$ independent configuration variables $\xi^{i}$. Specifically we assume the complexified description can be related to an alternative one in terms of a real Lagrangian $L_{\xi}\left(\xi^{1}, \dot{\xi}^{1}, \ldots, \xi^{n}, \dot{\xi}^{n}\right)$ through

$$
\begin{aligned}
& L\left(x, \dot{x}, \ldots, x^{(n)}\right)+\frac{d f}{d t}=L_{\xi}\left(\xi^{1}, \dot{\xi}^{1}, \ldots, \xi^{n}, \dot{\xi}^{n}\right), \\
& f=f\left(x, \ldots, x^{(n)}\right), \\
& \xi^{i}=\xi^{i}\left(x, \ldots, x^{(n)}\right), \quad i=1, \ldots, n .
\end{aligned}
$$

Here $\xi^{i}, i=1, \ldots, n$ are real coordinates and only their first order time derivatives $\dot{\xi}^{i}$ enter in $L_{\xi}$ in a quadratic form. In general $f$ will be complex. ii) The second point is that the transformation (2.4) will impose reality conditions on the $x^{(k)}$, with $k=0, \ldots, n$. This implies, in particular, that terms in the potential of higher order than quadratic must be real functions of the variables, i.e., under the analytic continuation, we must consider only the real part of the interaction, for example powers like $x^{4}$ should be replaced by $\left(x x^{\dagger}\right)^{2}$. This is not necessary for the quadratic terms, since our transformation incorporates them properly. We must notice that strictly speaking, our system is different from the original one, since we have analytically continued the $x$ variable. However, the reality conditions allow us to construct a properly well defined problem, with the same number of the original degrees of freedom together with the same classical equation for the original variable $x$.

\subsection{Canonical transformation and boundary conditions}

One of the interesting properties of our nonlocal transformation (2.4) is that in the HamiltonianOstrogradsky formalism [31], this transformation corresponds to a local canonical transformation. To see this, we observe that in this formalism the corresponding phase space will be of dimension $2 n$, so the configuration space is extended to $Q_{0}=x, Q_{1}=\dot{x}, \ldots, Q_{n-1}=x^{(n-1)}$, together with the momenta

$$
\Pi_{i}=\sum_{j=0}^{n-i-1}\left(-\frac{d}{d t}\right)^{j} \frac{\partial L}{\partial q^{(i+j+1)}}, \quad i=0, \ldots, n-1 .
$$

Thus, the transformation (2.4) only depends on the indicated phase space variables,

$$
\xi^{i}\left(x, \ldots, x^{(n)}\right)=\xi^{i}\left(Q_{0}, Q_{1}, \ldots, Q_{n-1}, \Pi_{n-1}\right),
$$

in which only the last momentum $\Pi_{n-1}$ appears. We also have the corresponding transformation for the momenta,

$$
P_{i}=P_{i}\left(Q_{0}, Q_{1}, \ldots, Q_{n-1}, \Pi_{0}, \ldots, \Pi_{n-1}\right),
$$

given directly from the generating function (2.3). The transformations (2.6), (2.7) will be canonical in an extended phase space because the Lagrangians $L$ and $L_{\xi}$ differ only by a total derivative that depends on the coordinates and momenta. We must remark that the transformations (2.6), 
(2.7) should be complex, because an analytic continuation of the original variables was required. However, these transformations define exactly the reality conditions, since we enforce that the $2 n$ variables $\left(\xi^{i}, P_{i}\right)$ will be real and, in consequence, also define the integration contour that must be used to quantize the system.

To build the explicit form of the canonical transformation that we are using, we consider the equivalent of the expression (2.2) in Hamiltonian form,

$$
\Pi_{i} \dot{Q}^{i}-\mathcal{H}(Q, \Pi)+\frac{d f(Q, \Pi)}{d t}=P_{i} \dot{\xi}^{i}-H(\xi, P) .
$$

From the comparison of independent terms we find

$$
\frac{\partial f}{\partial Q^{i}}=P_{j} \frac{\partial \xi^{j}}{\partial Q^{i}}-\Pi_{i}, \quad \frac{\partial f}{\partial \Pi_{i}}=P_{j} \frac{\partial \xi^{j}}{\partial \Pi_{i}}, \quad \mathcal{H}=H
$$

These equations prescribe the $2 n$ partial derivatives of the generating function $f(Q, \Pi)$ and the Hamiltonians are equal due to the fact that the transformation is time independent.

Now, to analyze the boundary conditions that are imposed by our complex canonical transformation, we make the variation of the left hand side of expression (2.8) and we observe that the boundary condition is associated with the total derivative

$$
\frac{d}{d t}\left(\left(\Pi_{i}+\frac{\partial f}{\partial Q^{i}}\right) \delta Q^{i}+\frac{\partial f}{\partial \Pi_{i}} \delta \Pi_{i}\right)
$$

which dictates the appropriate combinations of the variations $\delta Q^{i}$ and $\delta \Pi_{i}$ to be fixed at the boundaries. Using the equations $(2.9)$ we can show that the above expression is indeed $\frac{d}{d t}\left(P_{i} \delta \xi^{i}\right)$, in terms of the variation of the $\xi^{i}$. So, the variables that we need to fix on the boundary are those functions of $Q^{i}$ and $P_{i}$ defined by the $\xi^{i}$ 's.

\section{Reality conditions and the complex oscillator}

Let us start by reviewing the example of a modified harmonic oscillator subject to a complex canonical transformation $[24,25]$. This has also been studied in a form that exploits the $\mathcal{P} \mathcal{T}$ symmetry in the framework of the non-Hermitian Hamiltonian approach [23, 32, 33, 34]. The strategy is based on the observation that the complex Lagrangian

$$
L_{\mathrm{C}}=\frac{\dot{q}^{2}}{2}-\frac{q^{2}}{2}-i \epsilon q \dot{q}
$$

where $\epsilon$ is a real parameter, becomes the one corresponding to the harmonic oscillator after

adding to it the total time derivative $\frac{d f_{\mathrm{C}}}{d t}, f_{\mathrm{C}}=i \epsilon \frac{q^{2}}{2}$. In the Hamiltonian description the canonical momentum is

$$
p=\frac{\partial L_{\mathrm{C}}}{\partial \dot{q}}=\dot{q}-i \epsilon q,
$$

so $p \in \mathbb{C}$ and should be quantized as a non-Hermitian operator.

\subsection{Hilbert space and reality conditions}

The quantum Hamiltonian becomes

$$
\hat{H}_{\mathrm{C}}=\frac{\hat{p}^{2}}{2}+\frac{\hat{q}^{2}}{2}-\frac{1}{2} \epsilon^{2} \hat{q}^{2}+\frac{i \epsilon}{2}\{\hat{p}, \hat{q}\}
$$


where $\{\hat{p}, \hat{q}\}=\hat{p} \hat{q}+\hat{q} \hat{p}$ so that a symmetric ordering is selected. Upon the canonical transformation $\hat{p}=\hat{P}-i \epsilon \hat{Q}, \hat{q}=\hat{Q}$, with $\hat{P}=\frac{d \hat{Q}}{d t}$, the Hamiltonian (3.1) becomes the standard one of the harmonic oscillator $\hat{H}_{\mathrm{HO}}=\frac{\hat{P}^{2}}{2}+\frac{\hat{Q}^{2}}{2}$, which is Hermitian whenever $\hat{Q}$ and $\hat{P}$ are.

The coordinate representation

$$
\hat{q} \psi(q)=q \psi(q), \quad \hat{p} \psi(q)=-i \frac{d \psi}{d q}(q),
$$

leads to a non-Hermitian form of the Hamiltonian (3.1) with the usual scalar product ${ }^{1}$. The corresponding Schrödinger equation becomes

$$
\psi^{\prime \prime}(q)-2 \epsilon q \psi^{\prime}(q)-\left(\left(1-\epsilon^{2}\right) q^{2}+\epsilon-2 E\right) \psi(q)=0 .
$$

Hence the eigenvalue problem for (3.1) can be related to that of the harmonic oscillator by using the wave functions of the latter $\varphi_{n}=N_{n} \mathrm{e}^{-\frac{q^{2}}{2}} H_{n}(q)$ and defining

$$
\psi_{n}(q):=\mathrm{e}^{\frac{\epsilon q^{2}}{2}} \varphi_{n}, \quad E_{n}=n+\frac{1}{2}, \quad n=0,1,2 \ldots
$$

A tedious but otherwise direct calculation shows that the eigenfunctions $\psi_{n}$ do not have positive norm in the Hilbert space $\mathcal{H}_{0}=L^{2}(\mathbb{R}, d q)$. However, the classical role of $f_{C}$ as the generator of a canonical transformation motivates the introduction of a quantum transformation such that (see for example $[35,36]$ for a general discussion)

$$
\hat{H}_{\mathrm{HO}}=\mathrm{e}^{-\epsilon \frac{q^{2}}{2}} \hat{H}_{\mathrm{C}} \mathrm{e}^{\epsilon \frac{q^{2}}{2}} \text {. }
$$

This non-unitary canonical transformation changes the measure $d q$ to $d \mu=\mathrm{e}^{-\epsilon q^{2}} d q$. Now the Hilbert space $\mathcal{H}=L^{2}(\mathbb{R}, d \mu)$ ensures (3.2) have positive norm since

$$
\langle n \mid m\rangle_{\mu}:=\int d q \mathrm{e}^{-\epsilon q^{2}} \psi_{n}^{*} \psi_{m}=\int d q \varphi_{n}^{*} \varphi_{m}=:\langle n \mid m\rangle_{q}=\delta_{m n}
$$

where subscripts denote the appropriate measure for the correspondent Hilbert space. Moreover, the reality conditions

$$
\hat{q}^{\dagger}=\hat{q}, \quad \hat{p}^{\dagger}=\hat{p}+2 i \epsilon \hat{q}
$$

are automatically implemented in $\mathcal{H}$, where (3.1) is Hermitian [24].

It is illuminating to consider the Green's functions corresponding, respectively, to $\hat{H}_{\mathrm{C}}$ and $\hat{H}_{\mathrm{HO}}$. We recall they can be defined as

$$
\begin{aligned}
& G_{\mathrm{C}}^{\epsilon}\left(q_{2}, q_{1}, E\right):=\left\langle q_{2}\left|\frac{1}{E-\hat{H}_{\mathrm{C}}}\right| q_{1}\right\rangle, \\
& G_{\mathrm{HO}}\left(q_{2}, q_{1}, E\right):=\left\langle q_{2}\left|\frac{1}{E-\hat{H}_{\mathrm{HO}}}\right| q_{1}\right\rangle .
\end{aligned}
$$

Now on account of (3.3), we can rearrange (3.5) to relate it with (3.6) as follows

$$
\begin{aligned}
G_{\mathrm{C}}^{\epsilon}\left(q_{2}, q_{1}, E\right) & =\left\langle q_{2}\left|\frac{1}{E-\hat{\mathcal{O}}_{\epsilon}^{-1} \hat{H}_{\mathrm{HO}} \hat{\mathcal{O}}_{\epsilon}}\right| q_{1}\right\rangle=\left\langle q_{2}\left|\hat{\mathcal{O}}_{\epsilon}^{-1} \frac{1}{E-\hat{H}_{\mathrm{HO}}} \hat{\mathcal{O}}_{\epsilon}\right| q_{1}\right\rangle \\
& =\mathrm{e}^{+\epsilon\left(\frac{q_{2}^{2}}{2}-\frac{q_{1}^{2}}{2}\right)} G_{\mathrm{HO}}\left(q_{2}, q_{1}, E\right), \quad \hat{\mathcal{O}}_{\epsilon}:=\mathrm{e}^{-\epsilon \frac{\hat{q}^{2}}{2}},
\end{aligned}
$$

where in the final line we use that $\hat{q}$ acts diagonally on the basis $|q\rangle$ and its dual. Thus the standard harmonic oscillator Green's function, $G_{\mathrm{HO}}\left(q_{2}, q_{1}, E\right)$, is obtained from that corresponding to the complex oscillator, $G_{\mathrm{C}}^{\epsilon}\left(q_{2}, q_{1}, E\right)$, upon multiplying the former by $\mathrm{e}^{\epsilon\left(\frac{q_{2}^{2}}{2}-\frac{q_{1}^{2}}{2}\right)}$.

\footnotetext{
${ }^{1}$ Using $\hat{p}=-i \frac{d}{d q}+i \epsilon q$ would yield the usual Hamiltonian for the harmonic oscillator which, however, requires a modified inner product to be Hermitian.
} 


\subsection{The Schwinger quantum action principle}

We can use Schwinger's quantum action principle to derive some dynamical properties of the system [37]. For instance, we can calculate the propagator $\left\langle q_{2}, t_{2} \mid q_{1}, t_{1}\right\rangle$. We will work in the Heisenberg picture, where we adopt the notation

$$
\begin{array}{ll}
\left\langle q_{2}, t_{2}\right| q\left(t_{2}\right)=q_{2}\left\langle q_{2}, t_{2}\right|, & q\left(t_{1}\right)\left|q_{1}, t_{1}\right\rangle=q_{1}\left|q_{1}, t_{1}\right\rangle, \\
\left\langle p_{2}^{*}, t_{2}\right| p^{\dagger}\left(t_{2}\right)=p_{2}^{*}\left\langle p_{2}^{*}, t_{2}\right|, & p\left(t_{1}\right)\left|p_{1}, t_{1}\right\rangle=p_{1}\left|p_{1}, t_{1}\right\rangle,
\end{array}
$$

To achieve our purpose, we first calculate the total variation of $\left\langle q_{2}, t_{2} \mid q_{1}, t_{1}\right\rangle$, given by

$$
\delta\left\langle q_{2}, t_{2} \mid q_{1}, t_{1}\right\rangle=i\left\langle q_{2}, t_{2}\left|G_{q_{2}}-G_{q_{1}}-H \delta T\right| q_{1}, t_{1}\right\rangle,
$$

where $T=t_{2}-t_{1}$, and the generators $G_{q_{i}}$ are given by

$$
G_{q_{2}}=p\left(t_{2}\right) \delta q_{2}, \quad G_{q_{1}}=p\left(t_{1}\right) \delta q_{1} .
$$

Starting from the Hamiltonian operator (3.1), we get the following Heisenberg operator equations

$$
\begin{aligned}
& \dot{q}(t)=p(t)+i \epsilon q(t), \\
& \dot{p}(t)=-\left(1-\epsilon^{2}\right) q(t)-i \epsilon p(t) .
\end{aligned}
$$

Fixing the final and initial conditions at $t_{2}$ and $t_{1}$ as $q\left(t_{2}\right)$ and $q\left(t_{1}\right)$, we obtain the solutions

$$
\begin{aligned}
& q(t)=A \cos (t)+B \sin (t), \\
& p(t)=(B-i \epsilon A) \cos (t)-(i \epsilon B+A) \sin (t),
\end{aligned}
$$

where the coefficients $A$ and $B$ are given by

$$
\begin{aligned}
& A=\frac{1}{\sin (T)}\left(q\left(t_{1}\right) \sin \left(t_{2}\right)-q\left(t_{2}\right) \sin \left(t_{1}\right)\right), \\
& B=\frac{1}{\sin (T)}\left(q\left(t_{2}\right) \cos \left(t_{1}\right)-q\left(t_{1}\right) \cos \left(t_{2}\right)\right) .
\end{aligned}
$$

In this way, we can express $p\left(t_{2}\right)$ and $p\left(t_{1}\right)$ in terms of the initial and final conditions $q\left(t_{1}\right)$ and $q\left(t_{2}\right)$, getting

$$
\begin{aligned}
& p\left(t_{1}\right)=\frac{1}{\sin (T)}\left(-(i \epsilon \sin (T)+\cos (T)) q\left(t_{1}\right)+q\left(t_{2}\right)\right), \\
& p\left(t_{2}\right)=\frac{1}{\sin (T)}\left(-(i \epsilon \sin (T)-\cos (T)) q\left(t_{2}\right)-q\left(t_{1}\right)\right) .
\end{aligned}
$$

With this relations, one can also calculate the commutator

$$
\left[q\left(t_{1}\right), q\left(t_{2}\right)\right]=i \sin (T) .
$$

Using all of the above, we can write the Hamiltonian as

$$
H=\frac{1}{2 \sin ^{2}(T)}\left(q^{2}\left(t_{1}\right)+q^{2}\left(t_{2}\right)-2 \cos (T) q\left(t_{2}\right) q\left(t_{1}\right)\right)-\frac{i}{2} \frac{\cos (T)}{\sin (T)} .
$$

Finally, from the equations (3.8), (3.9) we get:

$$
\delta\left\langle q_{2}, t_{2} \mid q_{1}, t_{1}\right\rangle=i\left\langle q_{2}, t_{2}\right|\left\{\left\{\frac{1}{\sin (T)}\left(\cos (T) q_{2}-q_{1}\right)-i \epsilon q_{2}\right\} \delta q_{2}\right.
$$




$$
\begin{aligned}
& +\left\{\frac{1}{\sin (T)}\left(\cos (T) q_{1}-q_{2}\right)+i \epsilon q_{1}\right\} \delta q_{1} \\
& \left.-\left\{\frac{1}{2 \sin ^{2}(T)}\left(q_{1}^{2}+q_{2}^{2}-2 \cos (T) q_{2} q_{1}\right)-\frac{i}{2} \frac{\cos (T)}{\sin (T)}\right\} \delta T\right\}\left|q_{1}, t_{1}\right\rangle \\
= & i\left\langle q_{2}, t_{2}\right| \delta\left\{\frac{1}{2 \sin (T)}\left(\left(q_{2}^{2}+q_{1}^{2}\right) \cos (T)-2 q_{2} q_{1}\right)\right. \\
& \left.-\frac{i \epsilon}{2}\left(q_{2}^{2}-q_{1}^{2}\right)-i \ln \left(\frac{1}{\sqrt{\sin (T)}}\right)\right\}\left|q_{1}, t_{1}\right\rangle .
\end{aligned}
$$

Integrating we obtain

$$
\begin{aligned}
\left\langle q_{2}, t_{2} \mid q_{1}, t_{1}\right\rangle= & \frac{1}{\sqrt{2 \pi i \sin (T)}} \exp \left\{\frac{i}{2 \sin (T)}\left\{\left(q_{2}^{2}+q_{1}^{2}\right) \cos (T)-2 q_{2} q_{1}\right\}\right\} \\
& \times \exp \left\{\frac{\epsilon}{2}\left(q_{2}^{2}-q_{1}^{2}\right)\right\}
\end{aligned}
$$

which is consistent with equation (3.7). Using the same procedure we now wish to obtain the quantity $\left\langle p_{2}^{*}, t_{2} \mid p_{1}, t_{1}\right\rangle$. Again, we begin by calculating its variation

$$
\delta\left\langle p_{2}^{*}, t_{2} \mid p_{1}, t_{1}\right\rangle=\left\langle p_{2}^{*}, t_{2}\left|G_{p_{2}^{*}}-G_{p_{1}}-H \delta T\right| p_{1}, t_{1}\right\rangle,
$$

where now

$$
G_{p_{2}^{*}}=-q\left(t_{2}\right) \delta p_{2}^{*}, \quad G_{p_{1}}=q\left(t_{1}\right) \delta p_{1} .
$$

We now need to fix the initial and final conditions at $t_{1}$ and $t_{2}$ as $p\left(t_{1}\right)$ and $p^{\dagger}\left(t_{2}\right)$. Thus, again we get (3.10) and (3.11) as solutions to the Heisenberg equations, but now with coefficients $A$ and $B$ given by

$$
\begin{aligned}
A= & \frac{1}{\left(\left(\epsilon^{2}+1\right) \sin (T)-2 i \epsilon \cos (T)\right)} \\
& \times\left\{\left(\cos \left(t_{2}\right)+i \epsilon \sin \left(t_{2}\right)\right) p\left(t_{1}\right)-\left(\cos \left(t_{1}\right)-i \epsilon \sin \left(t_{1}\right)\right) p^{\dagger}\left(t_{2}\right)\right\}, \\
B= & \frac{1}{\left(\left(\epsilon^{2}+1\right) \sin (T)-2 i \epsilon \cos (T)\right)} \\
& \times\left\{\left(\sin \left(t_{2}\right)-i \epsilon \cos \left(t_{2}\right)\right) p\left(t_{1}\right)-\left(i \epsilon \cos \left(t_{1}\right)+\sin \left(t_{1}\right)\right) p^{\dagger}\left(t_{2}\right)\right\} .
\end{aligned}
$$

In this way, we can express $q\left(t_{1}\right)$ and $q\left(t_{2}\right)$ in terms of the initial and final conditions $p\left(t_{1}\right)$ and $p^{\dagger}\left(t_{2}\right)$. The result is

$$
\begin{aligned}
& q\left(t_{1}\right)=\frac{1}{\left(\left(\epsilon^{2}+1\right) \sin (T)-2 i \epsilon \cos (T)\right)}\left\{(\cos (T)+i \epsilon \sin (T)) p\left(t_{1}\right)-p^{\dagger}\left(t_{2}\right)\right\}, \\
& q\left(t_{2}\right)=\frac{1}{\left(\left(\epsilon^{2}+1\right) \sin (T)-2 i \epsilon \cos (T)\right)}\left\{p\left(t_{1}\right)-(\cos (T)+i \epsilon \sin (T)) p^{\dagger}\left(t_{2}\right)\right\} .
\end{aligned}
$$

One can also calculate the commutator

$$
\left[p\left(t_{1}\right), p^{\dagger}\left(t_{2}\right)\right]=i\left(\left(\epsilon^{2}+1\right) \sin (T)-2 i \epsilon \cos (T)\right) .
$$

Using all of the above, we find the following expression for $H$ in terms of the operators $p^{\dagger}\left(t_{1}\right)$ and $p\left(t_{0}\right)$

$$
H=\frac{1}{2\left(\left(\epsilon^{2}+1\right) \sin (T)-2 i \epsilon \cos (T)\right)^{2}}
$$




$$
\begin{aligned}
& \times\left\{\left(1-\epsilon^{2}\right)\left(p^{2}\left(t_{1}\right)+p^{\dagger 2}\left(t_{2}\right)\right)-2\left(\left(\epsilon^{2}+1\right) \cos (T)+2 i \epsilon \sin (T)\right) p^{\dagger}\left(t_{2}\right) p\left(t_{1}\right)\right\} \\
& -\frac{i}{2} \frac{\left(\left(\epsilon^{2}+1\right) \cos (T)+2 i \epsilon \sin (T)\right)}{\left(\left(\epsilon^{2}+1\right) \sin (T)-2 i \epsilon \cos (T)\right)} .
\end{aligned}
$$

Since $p^{\dagger}\left(t_{2}\right)$ and $p\left(t_{1}\right)$ act according to equation (3.9) we get

$$
\begin{aligned}
\delta\left\langle p_{2}^{*},\right. & t_{2}\left|p_{1}, t_{1}\right\rangle=i\left\langle p_{2}^{*}, t_{2}\right|\left\{\frac{1}{\left(\left(\epsilon^{2}+1\right) \sin (T)-2 i \epsilon \cos (T)\right)}\right. \\
& \times\left\{\left((\cos (T)+i \epsilon \sin (T)) p_{2}^{*}-p_{1}\right) \delta p_{2}^{*}+\left((\cos (T)+i \epsilon \sin (T)) p_{1}-p_{2}^{*}\right) \delta p_{1}\right\} \\
& -\left(\frac { 1 } { 2 ( ( \epsilon ^ { 2 } + 1 ) \operatorname { s i n } ( T ) - 2 i \epsilon \operatorname { c o s } ( T ) ) ^ { 2 } } \left\{\left(1-\epsilon^{2}\right)\left(p_{1}^{2}+p_{2}^{* 2}\right)-2\left(\left(\epsilon^{2}+1\right) \cos (T)\right.\right.\right. \\
& \left.\left.\left.+2 i \epsilon \sin (T)) p_{2}^{*} p_{1}\right\}-\frac{i}{2} \frac{\left(\left(\epsilon^{2}+1\right) \cos (T)+2 i \epsilon \sin (T)\right)}{\left(\left(\epsilon^{2}+1\right) \sin (T)+2 i \epsilon \cos (T)\right)}\right) \delta T\right\}\left|p_{1}, t_{1}\right\rangle \\
& =i\left\langle p_{2}^{*}, t_{2}\right| \delta\left\{\frac{1}{2\left(\left(\epsilon^{2}+1\right) \sin (T)-2 i \epsilon \cos (T)\right)}\left\{(\cos (T)+i \epsilon \sin (T))\left(p_{2}^{* 2}+p_{1}^{2}\right)-2 p_{2}^{*} p_{1}\right\}\right. \\
& \left.-i \ln \left(\frac{1}{\sqrt{\left(\epsilon^{2}+1\right) \sin (T)-2 i \epsilon \cos (T)}}\right)\right\}\left|p_{1}, t_{1}\right\rangle .
\end{aligned}
$$

Integration produces

$$
\begin{aligned}
\left\langle p_{2}^{*}, t_{2} \mid p_{1}, t_{1}\right\rangle= & \frac{C}{\sqrt{\left(\epsilon^{2}+1\right) \sin (T)-2 i \epsilon \cos (T)}} \\
& \times \exp \left\{\frac{i}{2} \frac{\left((\cos (T)+i \epsilon \sin (T))\left(p_{2}^{* 2}+p_{1}^{2}\right)-2 p_{2}^{*} p_{1}\right)}{\left(\left(\epsilon^{2}+1\right) \sin (T)-2 i \epsilon \cos (T)\right)}\right\},
\end{aligned}
$$

where $C$ is a normalization constant. We note that

$$
\lim _{t_{2} \rightarrow t_{1}}\left\langle p_{2}^{*}, t_{2} \mid p_{1}, t_{1}\right\rangle=C \sqrt{-\frac{1}{2 i \epsilon}} \exp \left\{-\frac{1}{4 \epsilon}\left(p_{1}-p_{2}^{*}\right)^{2}\right\} .
$$

Furthermore, in the limit $\epsilon \rightarrow 0$, all eigenvalues $p$ cease to be complex, so in the double limit we must have

$$
\lim _{\epsilon \rightarrow 0}\left\langle p_{2}^{*}, t_{2} \rightarrow t_{1} \mid p_{1}, t_{1}\right\rangle=\delta\left(p_{2}-p_{1}\right) .
$$

Fixing the constant $C$ accordingly we obtain

$$
\begin{aligned}
\left\langle p_{2}^{*}, t_{2} \mid p_{1}, t_{1}\right\rangle= & \frac{1}{\sqrt{2 \pi i}} \frac{1}{\sqrt{\left(\epsilon^{2}+1\right) \sin (T)-2 i \epsilon \cos (T)}} \\
& \times \exp \left\{\frac{i}{2} \frac{\left((\cos (T)+i \epsilon \sin (T))\left(p_{2}^{* 2}+p_{1}^{2}\right)-2 p_{2}^{*} p_{1}\right)}{\left(\left(\epsilon^{2}+1\right) \sin (T)-2 i \epsilon \cos (T)\right)}\right\} .
\end{aligned}
$$

Next we compare the standard harmonic oscillator propagator with that obtained in equation (3.13). The bracket corresponding to the change of basis is readily shown to be

$$
\langle P \mid p\rangle=\sqrt{\frac{1}{2 \pi \epsilon}} \mathrm{e}^{-\frac{1}{2 \epsilon}(p-P)^{2}},
$$


which allow us to relate the propagator expressed in terms of Hermitian variables, $(\hat{P}, \hat{Q})$, with that written in terms of non-Hermitian ones, $(\hat{p}, \hat{q})$, as follows

$$
\left\langle p^{*}, t_{2} \mid p^{\prime}, t_{1}\right\rangle=\frac{1}{2 \pi \epsilon} \int d P d P^{\prime} \mathrm{e}^{-\frac{1}{2}\left(\left(p^{*}-P^{\prime}\right)^{2}+\left(p^{\prime}-P\right)^{2}\right)}\left\langle P^{\prime}, t_{2} \mid P, t_{1}\right\rangle .
$$

We have explicitly verified the above expression. The completeness relation in the non-Hermitian description results

$$
\int d^{2} p \mu\left(p, p^{*}\right)|p\rangle\left\langle p^{*}\right|=1, \quad \mu=\frac{1}{\sqrt{\pi \epsilon}} \mathrm{e}^{+\frac{1}{4 \epsilon}\left(p-p^{*}\right)^{2}} .
$$

\subsection{Path integral approach}

Consider the action for the modified harmonic oscillator in terms of the real and imaginary parts of $q$ and $p$,

$$
\begin{aligned}
S= & \int_{t_{1}}^{t_{2}} d t\left(p \dot{q}-\left(\frac{p^{2}}{2}+\frac{1}{2}\left(1-\epsilon^{2}\right) q^{2}+i \epsilon p q\right)\right)=\int_{t_{1}}^{t_{2}} d t\left(\left(p_{R}+i p_{I}\right)\left(\dot{q}_{R}+i \dot{q}_{I}\right)\right. \\
& \left.-\left(\frac{\left(p_{R}+i p_{I}\right)^{2}}{2}+\frac{1}{2}\left(1-\epsilon^{2}\right)\left(q_{R}+i q_{I}\right)^{2}+i \epsilon\left(p_{R}+i p_{I}\right)\left(q_{R}+i q_{I}\right)\right)\right) .
\end{aligned}
$$

To compute the propagator using the path integral of this system we need to select an integration contour given by the reality conditions (3.4), that in terms of real and imaginary parts is

$$
q_{I}=0, \quad p_{I}=-\varepsilon q_{R}
$$

These conditions are a pair of second class constraints in the framework of the Dirac's method of quantization [38, 39], so the path integral subjected to them is exactly the Senjanovic path integral [40]. In our case we obtain

$$
\left\langle q_{2}, t_{2} \mid q_{1}, t_{1}\right\rangle=\int D p_{R} D p_{I} D q_{R} D q_{I} \delta\left(q_{I}\right) \delta\left(p_{I}+\varepsilon q_{R}\right) \exp (i S),
$$

since the determinant of the Poisson bracket of the constraints is one. Integrating over the imaginary parts using the delta functionals results in

$$
\left\langle q_{2}, t_{2} \mid q_{1}, t_{1}\right\rangle=\int D p_{R} D q_{R} \exp \left(i \int_{t_{1}}^{t_{2}} d t\left(\left(p_{R}-i \epsilon q_{R}\right) \dot{q}_{R}-\left(\frac{p_{R}^{2}}{2}+\frac{q_{R}^{2}}{2}\right)\right)\right),
$$

Here we see that the path integral is reduced to the usual path integral of the harmonic oscillator plus a term that contributes only to the classical action, so finally the amplitude is

$$
\left\langle q_{2}, t_{2} \mid q_{1}, t_{1}\right\rangle=\left(\frac{1}{2 \pi i \hbar \sin T}\right)^{\frac{1}{2}} \exp \left(\frac{i}{2 \sin T}\left[\left(q_{1}^{2}+q_{2}^{2}\right) \cos T-2 q_{1} q_{2}\right]+\frac{\epsilon}{2}\left(q_{2}^{2}-q_{1}^{2}\right)\right),
$$

with $T=t_{2}-t_{1}$. In this way we recover the result (3.12).

Summarizing, we have provided a complex canonical transformation taking the complex harmonic oscillator (3.1) into the ordinary harmonic oscillator so that the non-Hermitian variables fulfill reality conditions in the Hilbert space $\mathcal{H}$ which are consistent with the canonical transformation. Let us observe that $\mathcal{P} \mathcal{T}$ symmetry has not been invoked in this approach. The states (3.2) possess positive definite norm in $\mathcal{H}$ and a unitary time evolution follows from the canonical transformation. 


\section{The modified PU oscillator as a second order theory}

Now we proceed with the HOTD model. Let us start with the PU oscillator Lagrangian

$$
L_{\mathrm{PU}}=-\frac{1}{2} \ddot{x}^{2}+\frac{\left(\omega_{1}^{2}+\omega_{2}^{2}\right)}{2} \dot{x}^{2}-\frac{\omega_{1}^{2} \omega_{2}^{2}}{2} x^{2},
$$

where we assume $\omega_{1}>\omega_{2} . L_{\mathrm{PU}}$ is connected to

$$
L_{\xi}=\frac{1}{2} \dot{\xi}_{1}^{2}-\frac{\omega_{1}^{2}}{2} \xi_{1}^{2}+\frac{1}{2} \dot{\xi}_{2}^{2}-\frac{\omega_{2}^{2}}{2} \xi_{2}^{2},
$$

for real $\xi_{i}, i=1,2$, by the following relations

$$
\begin{array}{ll}
L_{\mathrm{PU}}+\frac{d f}{d t}=L_{\xi}, & f=\dot{x} \ddot{x}, \\
\xi_{1}=i(a x+b \ddot{x}), & \xi_{2}=c x+b \ddot{x} .
\end{array}
$$

By choosing $a, b, c$ to be real we see that $x$ is necessarily complex. To accomplish (4.3), (4.4) the following values are obtained, for which the same sign should be used,

$$
\frac{a}{\omega_{2}^{2}}=b=\frac{c}{\omega_{1}^{2}}= \pm \frac{1}{\sqrt{\omega_{1}^{2}-\omega_{2}^{2}}}
$$

In other words, according to (4.3), $L_{\mathrm{PU}}$ in (4.1) fails to be the real $L_{\xi}$ in (4.2) only by a total time derivative. Note that $f$ will give rise to the corresponding canonical transformation once it is expressed in terms of phase space variables which we now derive. According to the Ostrogradsky method applied to (4.1) we get

$$
\Pi_{x}=\left(\omega_{1}^{2}+\omega_{2}^{2}\right) \dot{x}+\dddot{x}, \quad z=\dot{x}, \quad \Pi_{z}=-\ddot{x},
$$

so that $\left\{x, \Pi_{x}\right\}=1$ and $\left\{z, \Pi_{z}\right\}=1$. Clearly $f=-z \Pi_{z}$. The quantum Hamiltonian is

$$
\hat{H}_{\mathrm{PU}}=-\frac{1}{2} \hat{\Pi}_{z}^{2}-\frac{\omega_{1}^{2}+\omega_{2}^{2}}{2} \hat{z}^{2}+\hat{z} \hat{\Pi}_{x}+\frac{\omega_{1}^{2} \omega_{2}^{2}}{2} \hat{x}^{2}
$$

Using the afore mentioned transformation we have

$$
\begin{array}{ll}
\hat{x}=i b \hat{\xi}_{1}+b \hat{\xi}_{2}, & \hat{\Pi}_{x}=i a \hat{P}_{1}+c \hat{P}_{2}, \\
\hat{z}=i b \hat{P}_{1}+b \hat{P}_{2}, & \hat{\Pi}_{z}=i c \hat{\xi}_{1}+a \hat{\xi}_{2},
\end{array}
$$

where $\hat{P}_{i}, i=1,2$, are the canonical momenta conjugated to $\hat{\xi}_{i}$ obtained from (4.2). In terms of these Hermitian variables the Hamiltonian (4.6) takes the form

$$
\hat{H}_{\xi}=\frac{\hat{P}_{1}^{2}}{2}+\frac{\omega_{1}^{2}}{2} \hat{\xi}_{1}^{2}+\frac{\hat{P}_{2}^{2}}{2}+\frac{\omega_{2}^{2}}{2} \hat{\xi}_{2}^{2}
$$

So, starting from a Hamiltonian that is not bounded from below, extending the canonical variables to the complex plane and adding a total time derivative a well defined Hamiltonian in terms of $P_{i}$ and $\xi_{i}$ is obtained. Notice that in [16] it is acknowledged there exists a similarity transformation relating the original PU oscillator with a couple of independent harmonic oscillators similar to our case. Nevertheless a further transformation has to be supplied in [16] to obtain the right frequencies for the oscillators. 


\subsection{The modified PU Lagrangian in the unequal frequency case in the extended phase space}

Instead of performing the canonical analysis starting from equation (4.1), we will consider (4.3) which includes the additional time derivative. Namely we start from

$$
L_{\mathrm{T}}=\frac{1}{2} \ddot{x}^{2}+\frac{1}{2}\left(\omega_{1}^{2}+\omega_{2}^{2}\right) \dot{x}^{2}-\frac{1}{2} \omega_{1}^{2} \omega_{2}^{2} x^{2}+\dot{x} x^{(3)} .
$$

We first notice that $L_{\mathrm{T}}=L_{\mathrm{T}}\left(x, \dot{x}, \ddot{x}, x^{(3)}\right)$, so it is higher order than $L_{\mathrm{PU}}$. This also means that, according to Ostrogradsky's formalism, there will be a six-dimensional phase space with coordinates $(x, \dot{x}, \ddot{x})$ and momenta $\left(p_{0}, p_{1}, p_{2}\right)$ given by

$$
\begin{aligned}
& p_{0}=\left(\omega_{1}^{2}+\omega_{2}^{2}\right) \dot{x}+x^{(3)}, \\
& p_{1}=0 \\
& p_{2}=\dot{x}
\end{aligned}
$$

according to the general definitions given in equation (2.5). Now we notice that (4.11) and (4.12) are in fact primary constraints, which we write as

$$
\begin{aligned}
& \phi_{1}=p_{1}=0, \\
& \phi_{2}=p_{2}-\dot{x}=0 .
\end{aligned}
$$

The time evolution of the above constraints fixes the corresponding Lagrange multipliers making them second class constraints. In this way, the reduced phase space has dimension four. We will choose to express our Hamiltonian in terms of $\left(x, \ddot{x}, p_{0}, p_{2}\right)$. The resulting canonical Hamiltonian is given by

$$
H_{\mathrm{C}}=p_{0} \dot{x}+p_{1} \ddot{x}+p_{2} x^{(3)}-L_{\mathrm{T}}=p_{0} p_{2}-\frac{1}{2} \ddot{x}^{2}-\frac{1}{2}\left(\omega_{1}^{2}+\omega_{2}^{2}\right) p_{2}^{2}+\frac{1}{2} \omega_{1}^{2} \omega_{2}^{2} x^{2} .
$$

Next we construct the canonical structure in the Dirac formalism, accounting for the second class constraints. To do so, we use the Dirac brackets

$$
\{A, B\}^{*}=\{A, B\}-\left\{A, \phi_{\alpha}\right\} C^{\alpha \beta}\left\{\phi_{\beta}, B\right\}, \quad \alpha, \beta=1,2,
$$

where $C^{\alpha \beta}$ is a square antisymmetric matrix such that

$$
C^{\mu \alpha}\left\{\phi_{\alpha}, \phi_{\nu}\right\}=\delta_{\nu}^{\mu}
$$

Upon using equations (4.13) and (4.14) we obtain

$$
C^{\alpha \beta}=\left(\begin{array}{cc}
0 & -1 \\
1 & 0
\end{array}\right) .
$$

The resulting Dirac brackets are

$$
\left\{x, p_{0}\right\}^{*}=1, \quad\left\{\ddot{x}, p_{2}\right\}^{*}=1,
$$

and zero for any other combination. Next we rewrite the Lagrangian in the canonical formalism

$$
L_{\mathrm{T}}=\dot{x} p_{0}+\ddot{x} p_{1}+x^{(3)} p_{2}-H_{\mathrm{C}}
$$

and make the constraints (4.13), (4.14) strong obtaining

$$
L_{\mathrm{T}}=\dot{x} p_{0}+x^{(3)} p_{2}-H_{\mathrm{C}} .
$$


In order to make contact with the classical version of the Hamiltonian (4.6) we introduce the following canonically related new variables, leaving $x$ unchanged,

$$
\Pi_{x}=p_{0}, \quad z=p_{2}, \quad \Pi_{z}=-\ddot{x} .
$$

Using equation (4.15), we obtain as the only non-zero Dirac brackets

$$
\left\{x, \Pi_{x}\right\}^{*}=1, \quad\left\{z, \Pi_{z}\right\}^{*}=1 .
$$

In this way, we can consider the variables $\left\{x, z, \Pi_{x}, \Pi_{z}\right\}$ as a set of canonical variables in the Dirac formalism. Introducing the new variables in equation (4.16) we obtain

$$
L_{\mathrm{T}}=\dot{x} \Pi_{x}-\dot{\Pi}_{z} z-H_{\mathrm{C}},
$$

where $H_{\mathrm{C}}$ corresponds to the classical counterpart of $H_{\mathrm{PU}}$ given in equation (4.6). The above equation guarantees that $H_{\mathrm{PU}}$ is the correct Hamiltonian in the reduced physical space and imply that the correct boundary conditions at the end points are given by fixing $x$ and $\Pi_{z}$ in regard to the variational problem.

\subsection{The Pais-Uhlenbeck transformation in matrix form}

The complex canonical transformation between the real variables $\left\{\xi_{1}, \xi_{2}, P_{1}, P_{2}\right\}$ and the complex ones $\left\{x, z, \Pi_{x}, \Pi_{z}\right\}$, is given in matrix form by

$$
X=M \xi, \quad \xi=M^{-1} X,
$$

where $X, \xi$ and $M$ stand for

$$
X=\left(\begin{array}{c}
x \\
z \\
\Pi_{x} \\
\Pi_{z}
\end{array}\right), \quad \xi=\left(\begin{array}{c}
\xi_{1} \\
\xi_{2} \\
P_{1} \\
P_{2}
\end{array}\right), \quad M=\left(\begin{array}{cccc}
i b & b & 0 & 0 \\
0 & 0 & i b & b \\
0 & 0 & i a & c \\
i c & a & 0 & 0
\end{array}\right)
$$

Now, the matrix $M$ satisfies

$$
M^{T} \Omega M=\Omega,
$$

with

$$
\Omega=\left(\begin{array}{cccc}
0 & 0 & 1 & 0 \\
0 & 0 & 0 & 1 \\
-1 & 0 & 0 & 0 \\
0 & -1 & 0 & 0
\end{array}\right)
$$

where only the relation $b(c-a)=1$ was needed to prove equation (4.17). This means that the matrix $M$ belongs to the complex symplectic group $\operatorname{Sp}(2 n, \mathcal{C})$, with $n=2$. Since $\operatorname{Sp}(2 n, \mathcal{C})$ is a $2 n(2 n+1)$ parameter group, in our case $M$ should have 20 parameters in general. One notices that

$$
\operatorname{det}(M)=(b(c-a))^{2}=1,
$$

which verifies the unimodular character of the symplectic matrix. 


\subsection{Hilbert space}

We have related the Hamiltonians (4.6) and (4.9) by means of the complex canonical transformations (4.7), (4.8) and their inverse

$$
\begin{array}{ll}
\xi_{1}=i a x-i b \Pi_{z}, & \xi_{2}=c x-b \Pi_{z}, \\
P_{\xi_{1}}=-i c z+i b \Pi_{x}, & P_{\xi_{2}}=-a z+b \Pi_{x},
\end{array}
$$

where the coefficients $a, b$ and $c$ are given in (4.5). We notice that in this case the following relations are satisfied:

$$
a c=\omega_{1}^{2} \omega_{2}^{2} b^{2}, \quad b(c-a)=1 .
$$

At the quantum mechanical level, we fix the reality conditions by requiring that the operators $\left\{\xi_{1}, \xi_{2}, P_{\xi_{1}}, P_{\xi_{2}}\right\}$ are Hermitian. This in turn implies that:

$$
\begin{array}{ll}
x^{\dagger}=b(a+c) x-2 b^{2} \Pi_{z}, & z^{\dagger}=-b(a+c) z+2 b^{2} \Pi_{x}, \\
\Pi_{z}^{\dagger}=2 a c x-b(a+c) \Pi_{z}, & \Pi_{x}^{\dagger}=-2 a c z+b(a+c) \Pi_{x} .
\end{array}
$$

We will quantize the system in the basis $\left|x, \Pi_{z}\right\rangle$, so we propose the following operator realizations

$$
\hat{x}=x, \quad \hat{\Pi}_{z}=\Pi_{z},
$$

and

$$
\hat{\Pi}_{x}=-i \frac{\partial}{\partial x}, \quad \hat{z}=i \frac{\partial}{\partial \Pi_{z}} .
$$

Now, the inner product written in the basis $\left|x, \Pi_{z}\right\rangle$ can be expressed in general as

$$
\langle\phi, \psi\rangle=\int d^{2} x d^{2} \Pi_{z} \mu\left(x, \Pi_{z}\right) \phi^{*}\left(x, \Pi_{z}\right) \psi\left(x, \Pi_{z}\right),
$$

where

$$
d^{2} x=d x_{R} d x_{I}, \quad d^{2} \Pi_{z}=d \Pi_{z R} d \Pi_{z I} .
$$

Because of conditions (4.18) and (4.19), we should have in particular:

$$
\langle x \phi, \psi\rangle=\left\langle\phi,\left(b(a+c) x-2 b^{2} \Pi_{z}\right) \psi\right\rangle,
$$

and

$$
\left\langle\Pi_{z} \phi, \psi\right\rangle=\left\langle\phi,\left(2 a c x-b(a+c) \Pi_{z}\right) \psi\right\rangle .
$$

or

$$
\begin{aligned}
& \int d^{2} x d^{2} \Pi_{z} \mu x^{*} \phi^{*} \psi=\int d^{2} x d^{2} \Pi_{z} \mu \phi^{*}\left\{b(a+c) x-2 b^{2} \Pi_{z}\right\} \psi, \\
& \int d^{2} x d^{2} \Pi_{z} \mu \Pi_{z}^{*} \phi^{*} \psi=\int d^{2} x d^{2} \Pi_{z} \mu \phi^{*}\left\{2 a c x-b(a+c) \Pi_{z}\right\} \psi .
\end{aligned}
$$

Separating $x$ and $\Pi_{z}$ in their real and imaginary parts inside the integral, and matching both equations, we conclude from (4.20) and (4.21) that:

$$
\begin{aligned}
& a x_{R}=b \Pi_{z R}, \\
& c x_{I}=b \Pi_{z I},
\end{aligned}
$$

which can be always accomplished if

$$
\mu\left(x, \Pi_{z}\right)=C \delta\left(a x_{R}-b \Pi_{z R}\right) \delta\left(c x_{I}-b \Pi_{z I}\right),
$$

where $C$ is a constant to be determined by some normalization condition. 


\subsection{The Schrödinger equation}

Let us consider the stationary Schödinger equation

$$
H_{M} \psi\left(x, \Pi_{z}\right)=E \psi\left(x, \Pi_{z}\right),
$$

or more explicitly, using the operator realizations given above

$$
-\frac{1}{2} \Pi_{z}^{2} \psi+\frac{1}{2}\left(\omega_{1}^{2}+\omega_{2}^{2}\right) \frac{\partial^{2} \psi}{\partial \Pi_{z}^{2}}+\frac{1}{2} \omega_{1}^{2} \omega_{2}^{2} x^{2} \psi+\frac{\partial^{2} \psi}{\partial x \partial \Pi_{z}}=E \psi .
$$

Using the relation between $\left\{\xi_{1}, \xi_{2}\right\}$ and $\left\{x, \Pi_{z}\right\}$, applying the chain rule

$$
\begin{aligned}
& \frac{\partial}{\partial x}=\sum_{i} \frac{\partial \xi_{i}}{\partial x} \frac{\partial}{\partial \xi_{i}}=i a \frac{\partial}{\partial \xi_{1}}+c \frac{\partial}{\partial \xi_{2}}, \\
& \frac{\partial}{\partial \Pi_{z}}=\sum_{i} \frac{\partial \xi_{i}}{\partial \Pi_{z}} \frac{\partial}{\partial \xi_{i}}=-i b \frac{\partial}{\partial \xi_{1}}-b \frac{\partial}{\partial \xi_{2}},
\end{aligned}
$$

and substituting we arrive at

$$
-\frac{1}{2} \frac{\partial^{2} \psi}{\partial \xi_{1}^{2}}-\frac{1}{2} \frac{\partial^{2} \psi}{\partial \xi_{2}^{2}}+\frac{\omega_{1}^{2}}{2} \xi_{1}^{2} \psi+\frac{\omega_{2}^{2}}{2} \xi_{2}^{2} \psi=E \psi,
$$

with regular solutions:

$$
\psi_{m, n}\left(\xi_{1}, \xi_{2}\right)=\exp \left\{-\frac{1}{2}\left(\omega_{1} \xi_{1}^{2}+\omega_{2}^{2} \xi_{2}^{2}\right)\right\} H_{m}\left(\sqrt{\omega_{1}} \xi_{1}\right) H_{n}\left(\sqrt{\omega_{2}} \xi_{2}\right) .
$$

Let us now consider the ground state, $m=n=0$

$$
\psi_{0,0}\left(\xi_{1}, \xi_{2}\right)=\exp \left\{-\frac{1}{2}\left(\omega_{1} \xi_{1}^{2}+\omega_{2}^{2} \xi_{2}^{2}\right)\right\}
$$

or written in terms of $x, \Pi_{z}$

$$
\psi_{0,0}\left(x, \Pi_{z}\right)=\exp \left\{\frac{1}{2}\left(\left(\omega_{1} a^{2}-\omega_{2} c^{2}\right) x^{2}+2 b\left(\omega_{2} c-\omega_{1} a\right) \Pi_{z} x+b^{2}\left(\omega_{1}-\omega_{2}\right) \Pi_{z}^{2}\right)\right\} .
$$

Separating $x$ and $\Pi_{z}$ in their real and imaginary parts we get

$$
\begin{aligned}
\psi_{0,0}\left(x, \Pi_{z}\right)= & \exp \left\{\frac { 1 } { 2 } \left(\left(\omega_{1} a^{2}-\omega_{2} c^{2}\right)\left(x_{R}^{2}-x_{I}^{2}\right)+2 b\left(\omega_{2} c-\omega_{1} a\right)\left(\Pi_{z R} x_{R}-\Pi_{z I} x_{I}\right)\right.\right. \\
& \left.\left.+b^{2}\left(\omega_{1}-\omega_{2}\right)\left(\Pi_{z R}^{2}-\Pi_{z I}^{2}\right)\right)\right\} \exp \left\{i \phi\left(x, \Pi_{z}\right)\right\},
\end{aligned}
$$

where $\phi\left(x, \Pi_{z}\right)$ is a real function of $x_{R}, x_{I}, \Pi_{z R}$ and $\Pi_{z I}$ and therefore is the imaginary part of $\psi_{0,0}$ written as a phase factor. It is not easy to see from (4.24) if the ground state function vanishes at $|x| \rightarrow \pm \infty,\left|\Pi_{z}\right| \rightarrow \pm \infty$. However, substituting the reality conditions (4.22), (4.23) we have

$$
\psi_{0,0}\left(z, \Pi_{z}\right)=\exp \left\{-\frac{\omega_{2}\left(\omega_{1}^{2}-\omega_{2}^{2}\right)}{2} x_{R}^{2}\right\} \exp \left\{-\frac{\omega_{1}\left(\omega_{1}^{2}-\omega_{2}^{2}\right)}{2} x_{I}^{2}\right\},
$$

where we verify that the ground state function indeed vanishes at infinity.

Finally, in the Appendix we compute the full propagator of the PU oscillator. 


\subsection{The modified PU Lagrangian in the equal frequency case as a third order system}

We now wish to follow the same procedure as in Subsection 4.1 for the restriction of the PU Lagrangian to the equal frequency case. Recapitulating, we begin from the corresponding equal frequency PU Lagrangian $\tilde{L}_{\mathrm{PU}}$ plus a total time derivative

$$
\tilde{L}_{\mathrm{T}}=\tilde{L}_{\mathrm{PU}}+\frac{d \tilde{f}}{d t}=-\frac{1}{2} \ddot{x}^{2}+\omega^{2} \dot{x}^{2}-\frac{1}{2} \omega^{4} x^{2}+\frac{d \tilde{f}}{d t} .
$$

The equations of motion arising from $\tilde{L}_{\mathrm{PU}}$ are

$$
0=x^{(4)}+2 \omega^{2} x^{(2)}+\omega^{4} x
$$

which are not affected by the total time derivative.

At the Lagrangian level the choice

$$
\tilde{f}=\frac{1}{2}\left(\ddot{x}-\omega^{2} x\right) \dot{x}
$$

allows to transform (4.25) into the two oscillators system

$$
\tilde{L}_{\mathrm{PU}}+\frac{d \tilde{f}}{d t}=\tilde{L}_{\xi}=\frac{1}{2} \dot{\xi}_{1}^{2}+\frac{1}{2} \dot{\xi}_{2}^{2}-\frac{1}{2} \Omega^{2}\left(\xi_{1}^{2}+\xi_{2}^{2}\right),
$$

by means of the nonlocal transformation

$$
\xi_{1}=i(a x+b \ddot{x}), \quad \xi_{2}=c x+d \ddot{x} .
$$

together with the conditions

$$
\omega^{2}=\Omega^{2}, \quad b^{2}=d^{2}, \quad c d-a b=1 / 2, \quad c^{2}-a^{2}=\omega^{2} .
$$

For simplicity we can take $d=b$, which gives us

$$
a=\omega^{2} b-\frac{1}{4 b}, \quad c=\omega^{2} b+\frac{1}{4 b} .
$$

Then, in this case we have a free parameter in the transformation. Now, the total Lagrangian turns out to be

$$
\tilde{L}_{\mathrm{T}}=\frac{1}{2} \omega^{2} \dot{x}^{2}-\frac{1}{2} \omega^{4} x^{2}+\frac{1}{2} x^{(3)} \dot{x}-\frac{1}{2} \omega^{2} x \ddot{x},
$$

where $x^{(3)}=\dddot{x}$. According to Ostrogradsky, the canonical momenta conjugated to the coordinates $(x, \dot{x}, \ddot{x})$ are

$$
\begin{aligned}
& \tilde{p}_{0}=\frac{3}{2} \omega^{2} \dot{x}+x^{(3)}, \\
& \tilde{p}_{1}=-\frac{1}{2}\left(\omega^{2} x+\ddot{x}\right), \\
& \tilde{p}_{2}=\frac{1}{2} \dot{x},
\end{aligned}
$$

respectively. Again, (4.27) and (4.28) are primary constraints, which can be put as

$$
\phi_{1}=\tilde{p}_{1}+\frac{1}{2}\left(\omega^{2} x+\ddot{x}\right)=0, \quad \phi_{2}=\tilde{p}_{2}-\frac{1}{2} \dot{x}=0 .
$$


As in the previous case, the time evolution of them fixes the corresponding Lagrange multipliers, thus turning (4.29) into a set of second class constraints which reduce the dimension of the phase space from six to four. We choose to express our Hamiltonian in terms of variables $\left\{x, \ddot{x}, \tilde{p}_{0}, \tilde{p}_{2}\right\}$, eliminating $\dot{x}, \tilde{p}_{1}$ from the constraints. The result is

$$
\tilde{H}_{\mathrm{C}}=\tilde{p}_{0} \dot{x}+\tilde{p}_{1} \ddot{x}+\tilde{p}_{2} x^{(3)}-\tilde{L}_{\mathrm{T}}=2 \tilde{p}_{2}\left(\tilde{p}_{0}+\omega_{2} \tilde{p}_{2}\right)-4 \omega^{2} \tilde{p}_{2}^{2}+\frac{1}{2} \omega^{4} x^{2}-\frac{1}{2} \ddot{x}^{2} .
$$

A similar procedure as the one described in Subsection 4.1 yields the non-zero Dirac brackets

$$
\left\{x, \tilde{p}_{0}\right\}^{*}=1, \quad\left\{\ddot{x}, \tilde{p}_{0}\right\}^{*}=-\frac{1}{2} \omega^{2}, \quad\left\{\ddot{x}, \tilde{p}_{2}\right\}^{*}=\frac{1}{2},
$$

in the phase space $\left\{x, \ddot{x}, \tilde{p}_{0}, \tilde{p}_{2}\right\}$. In order to make contact with the original equal frequency PU problem, let us introduce the new variables $\tilde{x}, \tilde{z}, \tilde{\Pi}_{x}, \tilde{\Pi}_{z}$

$$
\tilde{x}=x, \quad \tilde{z}=2 \tilde{p}_{2}, \quad \tilde{\Pi}_{x}=\tilde{p}_{0}+\omega^{2} \tilde{p}_{2}, \quad \tilde{\Pi}_{z}=-\ddot{x},
$$

and proceed to calculate their Dirac brackets. The only non-zero results in the phase space $\left\{\tilde{x}, \tilde{z}, \tilde{\Pi}_{x}, \tilde{\Pi}_{z}\right\}$ are

$$
\left\{\tilde{x}, \tilde{\Pi}_{x}\right\}^{*}=1, \quad\left\{\tilde{z}, \tilde{\Pi}_{z}\right\}^{*}=1,
$$

The above indicates that we can consider the set of variables $\left\{\tilde{x}, \tilde{z}, \tilde{\Pi}_{x}, \tilde{\Pi}_{z}\right\}$ as canonical variables in the Dirac formalism. Substituting in $\tilde{H}_{\mathrm{C}}$ we get:

$$
\tilde{H}_{\mathrm{C}}=-\frac{1}{2} \tilde{\Pi}_{z}^{2}-\omega^{2} \tilde{z}^{2}+\tilde{\Pi}_{x} \tilde{z}+\frac{1}{2} \omega^{4} \tilde{x}^{2},
$$

which corresponds to the original Pais-Uhlenbeck Hamiltonian (4.6) in the equal-frequency case. As pointed out in references [17, 21], (4.30) is not diagonalizable and hence cannot be related by a similarity transformation to the two oscillator system.

However, the Pais-Uhlenbeck Hamiltonian in the equal frequencies case can be related to the Hamiltonian of two uncoupled oscillators

$$
H=\frac{1}{2}\left(P_{1}^{2}+P_{2}^{2}\right)+\frac{1}{2} \omega^{2}\left(\xi_{1}^{2}+\xi_{2}^{2}\right),
$$

through a transformation which is neither canonical nor of the similarity type, given by

$$
\left(\begin{array}{c}
x \\
z \\
\Pi_{x} \\
\Pi_{z}
\end{array}\right)=\left(\begin{array}{cccc}
i b & \sqrt{\frac{1}{\omega^{2}}+b^{2}} & 0 & 0 \\
0 & 0 & i d & d \\
0 & 0 & i\left(d \omega^{2}-\frac{1}{2 d}\right) & \left(d \omega^{2}+\frac{1}{2 d}\right) \\
i \omega^{2} \sqrt{\frac{1}{\omega^{2}}+b^{2}} & b \omega^{2} & 0 & 0
\end{array}\right)\left(\begin{array}{c}
\xi_{1} \\
\xi_{2} \\
P_{1} \\
P_{2}
\end{array}\right)
$$

or, conversely

$$
\left(\begin{array}{c}
\xi_{1} \\
\xi_{2} \\
P_{1} \\
P_{2}
\end{array}\right)=\left(\begin{array}{cccc}
i b \omega^{2} & 0 & 0 & -i \sqrt{\frac{1}{\omega^{2}}+b^{2}} \\
\omega^{2} \sqrt{\frac{1}{\omega^{2}}+b^{2}} & 0 & 0 & -b \\
0 & -i\left(d \omega^{2}+\frac{1}{2 d}\right) & i d & 0 \\
0 & -\left(d \omega^{2}-\frac{1}{2 d}\right) & d & 0
\end{array}\right)\left(\begin{array}{c}
x \\
z \\
\Pi_{x} \\
\Pi_{z}
\end{array}\right) .
$$

Even though we have not explored the quantization of the system in this case, we expect that the non existence of a similarity transformation between the PU oscillator and the two-oscillator system would be a signal of the non-diagonalizable property of the Hamiltonian related to the appearance of Jordan blocks, as discussed in [41, 17, 21]. 


\section{Discussion}

In this work we have proposed that a complex canonical transformation applied to a class of HOTD models with Lagrangians modified by a total time derivative can solve the drawbacks of such theories, namely negative norm states or ghosts, unbounded below Hamiltonian and non-unitary time evolution, keeping unmodified the original classical equations of motion. Such a transformation requires the original canonical variables to be analytically continued and further subjected to appropriate reality conditions so that the final canonical set matches the original number of real degrees of freedom. In particular while the final canonical variables $\xi^{i}$ are Hermitian the original ones $x^{(i)}$ fulfill some reality conditions dictated by the canonical transformation among such variables. The inner product in the original Hilbert space is defined in such way to reproduce the reality conditions. This idea has been applied previously to nonperturbative canonical quantum general relativity [24, 25] and the inclusion of reality conditions was mandatory to recover real gravity. Thus we have successfully provided a novel extension of such idea to the case of the PU oscillator as an example of HOTD models. An important point that we must clarify is that the analytic extension of the variables allow us to have a well defined transformation between the HOTD model and a second order theory. The interesting result is that both theories have the same degrees of freedom and are related by a complex transformation. So, our procedure to extend the original problem to the complex plane gives us the appropriate frame to find the mapping between both theories. The success of the proposed approach in the PU case motivates the study of its extension to other HOTD models, but this is beyond the scope of the present work.

To illustrate the procedure we have first reviewed the case of a complexified model that reduces to an harmonic oscillator. Although not of the HOTD type, this example exhibits negative norm states which are neatly eliminated using a complex canonical transformation. In this case we determined also the corresponding propagator and contrasted it with the usual one of the harmonic oscillator. To do so we adopted two different techniques: Schwinger's quantum action principle and the path integral approach, both of which led to the same result. Let us notice also that the path integral version of complex canonical variables for gravity has been studied [42].

Next we studied the PU oscillator (4.1) which is properly a HOTD model. After complexifying the original variables we constructed the complex canonical transformation (4.7), (4.8) which leads to a system of two decoupled harmonic oscillators (4.9) with just the frequencies $\omega_{1}$ and $\omega_{2}$ appearing in (4.1). The propagator of the model containing non-Hermitian variables was determined by adopting again Schwinger's quantum action principle. The equal frequency PU oscillator has been also described in the classical case. We show that there are some differences with the unequal frequency situation. First, we have here a free parameter in the transformation, perhaps a resemblance of the Barbero-Immirzi parameter in loop quantum gravity [29, 30]. Furthermore, in the reduced phase space there does not exist a similarity transformation between the PU oscillator with equal frequency and the second order oscillators. However, we found that this system is also mapped to a couple of harmonic oscillators, using a non-similarity type transformation.

Our approach to the PU oscillator is an alternative to that of [16] based on non-Hermitian but $\mathcal{P} \mathcal{T}$ symmetric Hamiltonians. We find ours has the following properties: (1) It is based on nonHermitian variables subjected to specific and well defined reality conditions arising from (4.7), (4.8) that make the higher-order Hamiltonian Hermitian in the appropriate Hilbert space with measure (A.3). (2) No $\mathcal{P} \mathcal{T}$ symmetry is required. (3) It works for at least the PU oscillator. (4) If we want to consider additional interaction terms as an anharmonic contribution, our proposal still works provided that we add the prescription $x^{4} \rightarrow\left(x x^{\dagger}\right)^{2}=b^{4}\left(\xi_{1}^{2}+\xi_{2}^{2}\right)^{2}$. This interaction term has the correct signs and the Hamiltonian is still bounded from below. (5) We must 
emphasize that the complexification of the original problem together with the reality conditions are a key ingredient in our approach which constitute the prescription that define our problem.

It is remarkable that this approach can be also applied to the case of a quadratic higher-order derivative free scalar field model. To see this notice that the following Lagrangian density

$$
\mathcal{L}=-\frac{1}{2}(\square \phi)^{2}-\frac{m_{1}^{2}+m_{2}^{2}}{2} \phi \square \phi-\frac{m_{1}^{2} m_{2}^{2}}{2} \phi^{2},
$$

is related to

$$
\mathcal{L}_{\psi}=-\frac{1}{2} \psi_{1} \square \psi_{1}-\frac{m_{1}^{2}}{2} \psi_{1}^{2}-\frac{1}{2} \psi_{2} \square \psi_{2}-\frac{m_{2}^{2}}{2} \psi_{2}^{2},
$$

by

$$
\begin{array}{ll}
\mathcal{L}+\partial_{\mu} f^{\mu}=\mathcal{L}_{\psi}, & f^{\mu}=-\square \phi \partial^{\mu} \phi, \\
\psi_{1}=i(a \phi+b \square \phi), & \psi_{2}=c \phi+b \square \phi,
\end{array}
$$

with $a, b, c$ having the same form as in the PU model (4.5) except for the replacing $\omega_{i} \rightarrow m_{i}$, $i=1,2$. Clearly (5.1) is ghost-free. Our approach seems promising even in the interacting case including a term of the type $\left(\phi \phi^{*}\right)^{2}=-\frac{1}{(c-a)^{4}}\left(\psi_{1}^{2}+\psi_{2}^{2}\right)^{2}[6,9]$, however all these generalizations deserve further study.

In closing we would like to mention some directions for further study along the lines proposed in the present work. Other physical mechanical models including at least third order derivatives might help to show the strength of the canonical approach here pursued. Also a crucial problem to come to terms with is field theory. The success in the simplest non interacting scalar field briefly described above requires full implementation at the quantum level. Finally, as it was mentioned in the introduction, higher order gravity has been one of the motivations to the present approach, which of course is a paramount challenge. Also it would be rather interesting to look for HOTD gauge field theories to test some aspects of the gravitational case.

\section{A Schwinger's quantum action principle and the PU oscillator}

In this appendix we compute the propagator of the PU oscillator, a result already obtained in [43]. To do this, we apply Schwinger's quantum action principle to calculate $\left\langle x_{2}^{*}, \Pi_{z 2}^{*}, t_{2}\right| x_{1}$, $\left.\Pi_{z 1}, t_{1}\right\rangle$. However, in this case we need to be more careful since our variables are complex. We will work in the Heisenberg picture, where the involved operators act as:

$$
\begin{array}{ll}
x\left(t_{1}\right)\left|x_{1}, \Pi_{z 1}, t_{1}\right\rangle=x_{1}\left|x_{1}, \Pi_{z 1}, t_{1}\right\rangle, \quad \Pi_{z}\left(t_{1}\right)\left|x_{1}, \Pi_{z 1}, t_{1}\right\rangle=\Pi_{z 1}\left|x_{1}, \Pi_{z 1}, t_{1}\right\rangle, \\
\left\langle x_{2}^{*}, \Pi_{z 2}^{*}, t_{2}\right| x^{\dagger}\left(t_{2}\right)=\left\langle x_{2}^{*}, \Pi_{z 2}^{*}, t_{2}\right| x_{2}^{*}, \quad\left\langle x_{2}^{*}, \Pi_{z 2}^{*}, t_{2}\right| \Pi_{z}^{\dagger}\left(t_{2}\right)=\left\langle x_{2}^{*}, \Pi_{z 2}^{*}, t_{2}\right| \Pi_{z 2}^{*} .
\end{array}
$$

As usual, we first calculate the total variation of the quantity $\left\langle x_{2}^{*}, \Pi_{z 2}^{*}, t_{2} \mid x_{1}, \Pi_{z 1}, t_{1}\right\rangle$, given by:

$$
\begin{aligned}
& \delta\left\langle x_{2}^{*}, \Pi_{z 2}^{*}, t \mid x_{1}, \Pi_{z 1}^{\prime \prime}, t_{1}\right\rangle \\
& =i\left\langle x_{2}^{*}, \Pi_{z 2}^{*}, t_{2}\left|\left\{\Pi_{x}^{\dagger}\left(t_{2}\right) \delta x_{2}^{*}-z^{\dagger}\left(t_{2}\right) \delta \Pi_{z 2}^{*}-\Pi_{x}\left(t_{1}\right) \delta x_{1}+z\left(t_{1}\right) \delta \Pi_{z 1}-H \delta T\right\}\right| x_{1}, \Pi_{z 1}, t_{1}\right\rangle,
\end{aligned}
$$

where $T=t_{2}-t_{1}$. Now, we must express $\left\{z, \Pi_{x}, z^{\dagger}(t), \Pi_{x}^{\dagger}(t)\right\}$ in terms of $\left\{x, \Pi_{z}, x^{\dagger}(t), \Pi_{z}^{\dagger}(t)\right\}$.

We solve the Heisenberg equations for the operators $x, z, \Pi_{x}$ and $\Pi_{z}$. This equations are:

$$
\begin{aligned}
& \dot{x}(t)=z(t), \\
& \dot{\Pi}_{x}(t)=-\omega_{1}^{2} \omega_{2}^{2} x(t), \\
& \dot{z}(t)=-\Pi_{z}(t),
\end{aligned}
$$




$$
\dot{\Pi}_{z}(t)=\left(\omega_{1}^{2}+\omega_{2}^{2}\right) z(t)-\Pi_{x}(t) .
$$

Uncoupling, we get the following equation for $x(t)$ :

$$
x^{(4)}(t)+\left(\omega_{1}^{2}+\omega_{2}^{2}\right) \ddot{x}(t)+\omega_{1}^{2} \omega_{2}^{2} x(t)=0,
$$

with general solution:

$$
x(t)=C_{1} e^{i \omega_{1} t}+C_{2} e^{-i \omega_{1} t}+C_{3} e^{i \omega_{2} t}+C_{4} e^{-i \omega_{2} t},
$$

while for the other operators we get:

$$
\begin{aligned}
& z(t)=i \omega_{1}\left(C_{1} e^{i \omega_{1} t}-C_{2} e^{-i \omega_{1} t}\right)+i \omega_{2}\left(C_{3} e^{i \omega_{2} t}-C_{4} e^{-i \omega_{2} t}\right), \\
& \Pi_{x}(t)=i \omega_{1} \omega_{2}^{2}\left(C_{1} e^{i \omega_{1} t}-C_{2} e^{-i \omega_{1} t}\right)+i \omega_{1}^{2} \omega_{2}\left(C_{3} e^{i \omega_{2} t}-C_{4} e^{-i \omega_{2} t}\right), \\
& \Pi_{z}(t)=\omega_{1}^{2}\left(C_{1} e^{i \omega_{1} t}+C_{2} e^{-i \omega_{1} t}\right)+\omega_{2}^{2}\left(C_{3} e^{i \omega_{2} t}-C_{4} e^{-i \omega_{2} t}\right) .
\end{aligned}
$$

We fix the constants $C_{i}$ 's in terms of initial conditions at time $t_{1}: x\left(t_{1}\right), \Pi_{z}\left(t_{1}\right)$ and final conditions at time $t_{2}: x^{\dagger}\left(t_{2}\right)$ and $\Pi_{z}^{\dagger}\left(t_{2}\right)$. To obtain such conditions, we use the reality properties (4.18), (4.19), valid at all times. In this way, we arrive to the following system of linear equations:

$$
\begin{aligned}
& x\left(t_{1}\right)=C_{1} e^{i \omega_{1} t_{1}}+C_{2} e^{-i \omega_{1} t_{1}}+C_{3} e^{i \omega_{2} t_{1}}+C_{4} e^{-i \omega_{2} t_{1}}, \\
& \Pi_{z}\left(t_{1}\right)=\omega_{1}^{2}\left(C_{1} e^{i \omega_{1} t_{1}}+C_{2} e^{-i \omega_{1} t_{1}}\right)+\omega_{2}^{2}\left(C_{3} e^{i \omega_{2} t_{1}}+C_{4} e^{-i \omega_{2} t_{1}}\right), \\
& x^{\dagger}\left(t_{2}\right)=-\left(C_{1} e^{i \omega_{1} t_{2}}+C_{2} e^{-i \omega_{1} t_{2}}\right)+\left(C_{3} e^{i \omega_{2} t_{2}}+C_{4} e^{-i \omega_{2} t_{2}}\right), \\
& \Pi_{z}^{\dagger}\left(t_{2}\right)=-\omega_{1}^{2}\left(C_{1} e^{i \omega_{1} t_{2}}+C_{2} e^{-i \omega_{1} t_{2}}\right)+\omega_{2}^{2}\left(C_{2} e^{i \omega_{2} t_{2}}+C_{4} e^{-\omega_{2} t_{2}}\right),
\end{aligned}
$$

Solving for the $C$ 's and substituting, we get the following expressions for $z\left(t_{1}\right), \Pi_{x}\left(t_{1}\right), z^{\dagger}\left(t_{2}\right)$ and $\Pi_{x}^{\dagger}\left(t_{2}\right)$ :

$$
\begin{aligned}
z\left(t_{1}\right)= & \frac{1}{\left(\omega_{1}^{2}-\omega_{2}^{2}\right) \sin \left(\omega_{1} T\right) \sin \left(\omega_{2} T\right)} \\
\times & \times\left\{-\omega_{1}^{2} \omega_{2} \sin \left(\omega_{1} T\right) \cos \left(\omega_{2} T\right)+\omega_{1} \omega_{2}^{2} \cos \left(\omega_{1} T\right) \sin \left(\omega_{2} T\right)\right\} x\left(t_{1}\right) \\
+ & \left\{\omega_{2} \sin \left(\omega_{1} T\right) \cos \left(\omega_{2} T\right)-\omega_{1} \cos \left(\omega_{1} T\right) \sin \left(\omega_{2} T\right)\right\} \Pi_{z}\left(t_{1}\right) \\
+ & \left.\left\{\omega_{1}^{2} \omega_{2} \sin \left(\omega_{1} T\right)+\omega_{1} \omega_{2}^{2} \sin \left(\omega_{2} T\right)\right\} x^{\dagger}\left(t_{2}\right)+\left\{-\omega_{2} \sin \left(\omega_{1} T\right)-\omega_{1} \sin \left(\omega_{2} T\right)\right\} \Pi_{z}^{\dagger}\left(t_{2}\right)\right\} \\
\Pi_{x}\left(t_{1}\right)= & \frac{1}{\left(\omega_{1}^{2}-\omega_{2}^{2}\right) \sin \left(\omega_{1} T\right) \sin \left(\omega_{2} T\right)} \\
& \times\left\{\left\{-\omega_{1}^{4} \omega_{2} \sin \left(\omega_{1} T\right) \cos \left(\omega_{2} T\right)+\omega_{1} \omega_{2}^{4} \cos \left(\omega_{1} T\right) \sin \left(\omega_{2} T\right)\right\} x\left(t_{1}\right)\right. \\
& +\left\{\omega_{1}^{2} \omega_{2} \sin \left(\omega_{1} T\right) \cos \left(\omega_{2} T\right)-\omega_{1} \omega_{2}^{2} \cos \left(\omega_{1} T\right) \sin \left(\omega_{2} T\right)\right\} \Pi_{z}\left(t_{1}\right) \\
& +\left\{\omega_{1}^{4} \omega_{2} \sin \left(\omega_{1} T\right)+\omega_{1} \omega_{2}^{4} \sin \left(\omega_{2} T\right)\right\} x^{\dagger}\left(t_{2}\right) \\
& \left.+\left\{-\omega_{1}^{2} \omega_{2} \sin \left(\omega_{1} t_{2}\right)-\omega_{1} \omega_{2}^{2} \sin \left(\omega_{2} T\right)\right\} \Pi_{z}^{\dagger}\left(t_{2}\right)\right\} \\
z^{\dagger}\left(t_{2}\right)= & \frac{1}{\left(\omega_{1}^{2}-\omega_{2}^{2}\right) \sin \left(\omega_{1} T\right) \sin \left(\omega_{2} T\right)}\left\{\left\{-\omega_{1}^{2} \omega_{2} \sin \left(\omega_{1} T\right)-\omega_{1} \omega_{2}^{2} \sin \left(\omega_{2} T\right)\right\} x\left(t_{1}\right)\right. \\
& +\left\{\omega_{2} \sin \left(\omega_{1} T\right)+\omega_{1} \sin \left(\omega_{2} T\right)\right\} \Pi_{z}\left(t_{1}\right) \\
& +\left\{\omega_{1}^{2} \omega_{2} \sin \left(\omega_{1} T\right) \cos \left(\omega_{2} T\right)-\omega_{1} \omega_{2}^{2} \cos \left(\omega_{1} T\right) \sin \left(\omega_{2} T\right)\right\} x^{\dagger}\left(t_{2}\right) \\
& \left.+\left\{-\omega_{2} \sin \left(\omega_{1} T\right) \cos \left(\omega_{2} T\right)+\omega_{1} \cos \left(\omega_{1} T\right) \sin \left(\omega_{2} T\right)\right\} \Pi_{z}^{\dagger}\left(t_{2}\right)\right\} \\
\Pi_{z}^{\dagger}\left(t_{2}\right)= & \frac{1}{\left(\omega_{1}^{2}-\omega_{2}^{2}\right) \sin \left(\omega_{1} T\right) \sin \left(\omega_{2} T\right)}\left\{\left\{-\omega_{1}^{4} \omega_{2} \sin \left(\omega_{1} T\right)-\omega_{1} \omega_{2}^{4} \sin \left(\omega_{2} T\right)\right\} x\left(t_{1}\right)\right. \\
& +\left\{\omega_{1}^{2} \omega_{2} \sin \left(\omega_{1} T\right)+\omega_{1} \omega_{2}^{2} \sin \left(\omega_{2} T\right)\right\} \Pi_{z}\left(t_{1}\right)
\end{aligned}
$$




$$
\begin{aligned}
& +\left\{\omega_{1}^{4} \omega_{2} \sin \left(\omega_{1} T\right) \cos \left(\omega_{2} T\right)-\omega_{1} \omega_{2}^{4} \cos \left(\omega_{1} T\right) \sin \left(\omega_{2} T\right)\right\} x^{\dagger}\left(t_{2}\right) \\
& \left.+\left\{-\omega_{1}^{2} \omega_{2} \sin \left(\omega_{1} T\right) \cos \left(\omega_{2} T\right)+\omega_{1} \omega_{2}^{2} \cos \left(\omega_{1} T\right) \sin \left(\omega_{2} T\right)\right\} \Pi_{z}^{\dagger}\left(t_{2}\right)\right\} .
\end{aligned}
$$

Using these results in the Hamiltonian (4.6) and in the total variation (A.1), we get finally

$$
\begin{aligned}
\left\langle x_{2}^{*}, \Pi_{z 2}^{*}, t_{2} \mid x_{1}, \Pi_{z 1}, t_{1}\right\rangle=\sqrt{\frac{1}{\sin \left(\omega_{1} T\right) \sin \left(\omega_{2} T\right)}} \exp \left\{\frac{i}{\left(\omega_{1}^{2}-\omega_{2}^{2}\right) \sin \left(\omega_{1} T\right) \sin \left(\omega_{2} T\right)}\right. \\
\quad \times\left\{\left\{\omega_{1}^{4} \omega_{2} \sin \left(\omega_{1} T\right) \cos \left(\omega_{2} T\right)-\omega_{1} \omega_{2}^{4} \cos \left(\omega_{1} T\right) \sin \left(\omega_{2} T\right)\right\}\left\{\frac{x_{1}^{2}}{2}+\frac{x_{2}^{* 2}}{2}\right\}\right. \\
\quad+\left\{\omega_{2} \sin \left(\omega_{1} T\right) \cos \left(\omega_{2} T\right)-\omega_{1} \cos \left(\omega_{1} T\right) \sin \left(\omega_{2} T\right)\right\}\left\{\frac{\Pi_{z 1}^{2}}{2}+\frac{\Pi_{z 2}^{* 2}}{2}\right\} \\
+\left\{\omega_{1}^{2} \omega_{2} \sin \left(\omega_{1} T\right)+\omega_{1} \omega_{2}^{2} \sin \left(\omega_{2} T\right)\right\}\left\{x_{2}^{*} \Pi_{z 1}+\Pi_{z 2}^{*} x_{1}\right\} \\
+\left\{-\omega_{1}^{2} \omega_{2} \sin \left(\omega_{1} T\right) \cos \left(\omega_{2} T\right)+\omega_{1} \omega_{2}^{2} \cos \left(\omega_{1} T\right) \sin \left(\omega_{2} T\right)\right\}\left\{x_{2}^{*} \Pi_{z 2}^{*}+x_{1} \Pi_{z 1}\right\} \\
+\left\{-\omega_{1}^{4} \omega_{2} \sin \left(\omega_{1} T\right)-\omega_{1} \omega_{2}^{4} \sin \left(\omega_{2} T\right)\right\}\left\{x_{2}^{*} x_{1}\right\} \\
\left.\left.\quad+\left\{-\omega_{2} \sin \left(\omega_{1} T\right)-\omega_{1} \sin \left(\omega_{2} T\right)\right\}\left\{\Pi_{z 2}^{*} \Pi_{z 1}\right\}\right\}\right\}
\end{aligned}
$$

or, for short:

$$
\begin{aligned}
& \left\langle x_{2}^{*}, \Pi_{z 2}^{*}, t_{2} \mid x_{1}, \Pi_{z 1}, t_{1}\right\rangle=Q \exp \left\{\frac { i } { D } \left\{F\left(x_{2}^{*} \Pi_{z 1}+x_{1} \Pi_{z 2}^{*}\right)+G \Pi_{z 1} \Pi_{z 2}^{*}\right.\right. \\
& \left.\left.+J\left(x_{2}^{*} \Pi_{z 2}^{*}+x_{1} \Pi_{z 1}\right)+K\left(\frac{\Pi_{z 2}^{* 2}+\Pi_{z 1}^{2}}{2}\right)+M x_{1} x_{2}^{*}+N\left(\frac{x_{2}^{* 2}+x_{1}^{2}}{2}\right)\right\}\right\},
\end{aligned}
$$

with $D, F, G, J, K, M, N, Q$, being the following functions of $T$ :

$$
\begin{aligned}
& D=\left(\omega_{1}^{2}-\omega_{2}^{2}\right) \sin \left(\omega_{1} T\right) \sin \left(\omega_{2} T\right), \\
& F=\omega_{1}^{2} \omega_{2} \sin \left(\omega_{1} T\right)+\omega_{1} \omega_{2}^{2} \sin \left(\omega_{2} T\right), \\
& G=-\omega_{2} \sin \left(\omega_{1} T\right)-\omega_{1} \sin \left(\omega_{2} T\right), \\
& J=-\omega_{1}^{2} \omega_{2} \sin \left(\omega_{1} T\right) \cos \left(\omega_{2} T\right)+\omega_{1} \omega_{2}^{2} \sin \left(\omega_{2} T\right) \cos \left(\omega_{1} T\right), \\
& K=\omega_{2} \sin \left(\omega_{1} T\right) \cos \left(\omega_{2} T\right)-\omega_{1} \sin \left(\omega_{2} T\right) \cos \left(\omega_{1} T\right), \\
& M=-\omega_{1}^{4} \omega_{2} \sin \left(\omega_{1} T\right)-\omega_{1} \omega_{2}^{4} \sin \left(\omega_{2} T\right), \\
& N=\omega_{1}^{4} \omega_{2} \sin \left(\omega_{1} T\right) \cos \left(\omega_{2} T\right)-\omega_{1} \omega_{2}^{4} \sin \left(\omega_{2} T\right) \cos \left(\omega_{1} T\right), \\
& Q=\sqrt{\frac{1}{\sin \left(\omega_{1} T\right) \sin \left(\omega_{2} T\right)}} .
\end{aligned}
$$

Just as in our first example the PU propagator (A.2) can be related to the one corresponding to the Hamiltonian (4.9) by using the change of basis

$$
\left\langle P_{1}, P_{2} \mid x, \Pi_{z}\right\rangle=\exp \left[\left(a x-b \Pi_{z}\right) P_{1}+\left(-i c x+i b \Pi_{z}\right) P_{2}\right] .
$$

The basis $\left|x, \Pi_{z}\right\rangle$ is complete with the measure

$$
d \mu_{P U}=\frac{d x_{R} d x_{I} d \Pi_{z R} d \Pi_{z I}}{(2 \pi)^{2}} \delta\left(b \Pi_{z R}-a x_{R}\right) \delta\left(b \Pi_{z I}-c x_{I}\right),
$$

where $x_{R}, x_{I}, \Pi_{z R}, \Pi_{z I}$ are real. 


\section{Acknowledgements}

This work was partially supported by the following grants: CONACyT-SEP 51132F, CONACyTSEP 47211-F, CONACyT-SEP 55310, DGAPA-UNAM IN109107 and a CONACyT sabbatical grant to HAMT. AD wishes also to acknowledge support from CONACyT.

\section{References}

[1] Thirring W., Regularization as a consequence of higher order equations, Phys. Rev. 77 (1950), 570.

[2] Pais A., Uhlenbeck G.E., On field theories with nonlocalized action, Phys. Rev. 79 (1950), 145-165.

[3] Heisenberg W., Lee model and quantisation of non linear field theories, Nuclear Phys. 4 (1957), 532-563.

[4] Stelle K.S., Renormalization of higher-derivative quantum gravity, Phys. Rev. D 16 (1977), 953-969.

[5] Tomboulis E.T., Unitarity in higher derivative quantum gravity, Phys. Rev. Lett. 52 (1984), 1173-1176.

[6] Hawking S.W., Hertog T., Living with ghosts, Phys. Rev. D 65 (2002), 103515, 8 pages, hep-th/0107088.

[7] Moeller N., Zwiebach B., Dynamics with infinitely many time derivatives and rolling tachyons, J. High Energy Phys. 2002 (2002), no. 10, 034, 39 pages, hep-th/0207107.

[8] Rivelles V.O., Triviality of higher derivative theories, Phys. Lett. B 577 (2003), 137-142, hep-th/0304073.

[9] Antoniadis I., Dudas E., Ghilencea D.M., Living with ghosts and their radiative corrections, Nuclear Phys. B 767(2007), 29-53, hep-th/0608094.

[10] Codello A., Percacci R., Fixed points of higher-derivative gravity, Phys. Rev. Lett. 97 (2006), 221301, 4 pages, hep-th/0607128.

[11] Berkovits N., New higher-derivative $R^{4}$ theorems, Phys. Rev. Lett. 98 (2007), 211601, 4 pages, hep-th/0609006.

[12] Jaen X., Llosa J., Molina A., A reduction of order two for infinite order Lagrangians, Phys. Rev. D 34 (1986), 2302-2311.

[13] Eliezer D.A., Woodard R.P., The problem of nonlocality in string theory, Nuclear Phys. B 325 (1989), 389-469.

[14] Cheng T.C., Ho P.M., Yeh M.C., Perturbative approach to higher derivative and nonlocal theories, Nuclear Phys. B 625 (2002), 151-165, hep-th/0111160.

[15] Simon J.Z., Higher derivative Lagrangians, non-locality, problems and solutions, Phys. Rev. D 41 (1990), 3720-3733.

[16] Bender C.M., Mannheim P.D., No-ghost theorem for the fourth-order derivative Pais-Uhlenbeck oscillator model, Phys. Rev. Lett. 100 (2008), 110402, 4 pages, arXiv:0706.0207.

[17] Bender C.M., Mannheim P.D., Exactly solvable $\mathcal{P} T$-symmetric Hamiltonian having no Hermitian counterpart, Phys. Rev. D 78 (2008), 025022, 20 pages, arXiv:0804.4190.

[18] Bender C.M., Mannheim P.D., Giving up the ghost, J. Phys. A: Math. Theor. 41 (2008), 304018, 7 pages, arXiv:0807.2607.

[19] Smilga A.V., Benign vs. malicious ghosts in higher-derivative theories, Nuclear Phys. B 706 (2005), 598-614, hep-th/0407231.

[20] Smilga A.V., Ghost-free higher-derivative theory, Phys. Lett. B 632 (2006), 433-438, hep-th/0503213.

[21] Smilga A.V., Comments on the dynamics of the Pais-Uhlenbeck oscillator, SIGMA 5 (2009), 017, 13 pages, arXiv:0808.0139.

[22] Smilga A.V., Exceptional points in quantum and classical dynamics, J. Phys. A: Math. Theor. 42 (2009), 095301, 9 pages, arXiv:0808.0575.

[23] Bender C.M., Making sense of non-Hermitian Hamiltonians, Rep. Progr. Phys. 70 (2007), 947-1018, hep-th/0703096.

[24] Ashtekar A., Lectures on nonperturbative canonical gravity, Advanced Series in Astrophysics and Cosmology, Vol. 6, World Scientific, Singapore, 1991.

[25] Ashtekar A., Mathematical problems of nonperturbative quantum general relativity, gr-qc/9302024.

[26] Thiemann T., Reality conditions inducing transforms for quantum gauge field theory and quantum gravity, Classical Quantum Gravity 13 (1996), 1383-1403, gr-qc/9511057. 
[27] Ashtekar A., A generalized Wick transform for gravity, Phys. Rev. D 53 (1996), 2865-2869, gr-qc/9511083.

[28] Montesinos M., Morales-Técotl H.A., Urrutia L.F., Vergara J.D., Complex canonical gravity and reality constraints, Gen. Relativity Gravitation 31 (1999), 719-723.

[29] Rovelli C., Quantum gravity, Cambridge University Press, Cambridge, 2004.

[30] Thiemann T., Modern canonical quantum general relativity, Cambridge University Press, Cambridge, 2007.

[31] Ostrogradsky M., Mémoires sur les équations différentielles relatives aux problèmes des isopérimètres, Mem. Acad. St. Petersbourg VI 4 (1850), 385-517.

[32] Swanson M.S., Transition elements for a non-Hermitian quadratic Hamiltonian, J. Math. Phys. 45 (2004), 585-601.

[33] Jones H.F., On pseudo-Hermitian Hamiltonians and their Hermitian counterparts, J. Phys. A: Math. Gen. 38 (2005), 1741-1746, quant-ph/0411171.

[34] Ivanov E.A., Smilga A.V., Cryptoreality of nonanticommutative Hamiltonians, J. High Energy Phys. 2007 (2007), no. 07, 036, 16 pages, hep-th/0703038.

[35] Anderson A., Canonical transformations in quantum mechanics, Ann. Physics 232 (1994), 292-331, hep-th/9305054.

[36] Anderson A., Quantum canonical transformations: physical equivalence of quantum theories, Phys. Lett. B 305 (1993), 67-70, hep-th/9302062.

[37] Schwinger J., Quantum mechanics, symbolism of atomic measurements, Springer-Verlag, Berlin, 2001.

[38] Henneaux M., Teitelboim C., Quantization of gauge systems, Princeton University Press, Princeton, 1992.

[39] Dirac P.A.M., Lectures on quantum mechanics, Belfast Graduate School of Science, New York, 1964.

[40] Senjanovic P., Path integral quantization of field theories with second class constraints, Ann. Physics 100 (1976), 227-261, Erratum, Ann. Physics 209 (1991), 248.

[41] Mannheim P.D., Davidson A., Dirac quantization of the Pais-Uhlenbeck fourth order oscillator, Phys. Rev. A 71 (2005), 042110, 9 pages, hep-th/0408104.

[42] Alexandrov S.Y., Vassilevich D.V., Path integral for the Hilbert-Palatini and Ashtekar gravity, Phys. Rev. D 58 (1998), 124029, 13 pages, gr-qc/9806001.

[43] Mannheim P.D., Solution to the ghost problem in fourth order derivative theories, Found. Phys. 37 (2007), 532-571, hep-th/0608154. 